\title{
Genetics of lymphatic anomalies
}

\author{
Pascal Brouillard,1 Laurence Boon,1,2 and Miikka Vikkula',2,3 \\ ${ }^{1}$ Laboratory of Human Molecular Genetics, de Duve Institute, ${ }^{2}$ Center for Vascular Anomalies, Division of Plastic Surgery, Cliniques Universitaires Saint-Luc, \\ and ${ }^{3}$ Walloon Excellence in Lifesciences and Biotechnology, Université catholique de Louvain, Brussels, Belgium.
}

\begin{abstract}
Lymphatic anomalies include a variety of developmental and/or functional defects affecting the lymphatic vessels: sporadic and familial forms of primary lymphedema, secondary lymphedema, chylothorax and chylous ascites, lymphatic malformations, and overgrowth syndromes with a lymphatic component. Germline mutations have been identified in at least 20 genes that encode proteins acting around VEGFR-3 signaling but also downstream of other tyrosine kinase receptors. These mutations exert their effects via the RAS/MAPK and the PI3K/AKT pathways and explain more than a quarter of the incidence of primary lymphedema, mostly of inherited forms. More common forms may also result from multigenic effects or post-zygotic mutations. Most of the corresponding murine knockouts are homozygous lethal, while heterozygotes are healthy, which suggests differences in human and murine physiology and the influence of other factors.
\end{abstract}

\section{Introduction}

The lymphatic system plays a crucial role in tissue homeostasis. Blunt-ended lymphatic capillaries collect extravasated fluid, transport it via collecting lymphatic vessels and the thoracic duct, and return it to the blood circulation at the level of the left subclavian vein. The lymph travels through lymph nodes for immune surveillance. Larger lymphatic vessels contain valves to orient the passive flow, driven by neighboring arterial pulsations and muscular contractions. Lymphatic anomalies result from defects in the development, maturation, or function of this system. These include lymphatic malformations (LMs), lymphedema, chylothorax and chylous ascites, and overgrowth syndromes with a lymphatic component, including the Klippel-Trenaunay-Weber, proteus, PTEN hamartoma tumor syndrome, and CLOVES (congenital lipomatous overgrowth, vascular malformations, epidermal nevi, and skeletal/spinal abnormalities) syndromes (1). Genetic studies and the generation of genetically modified mice have started to shed light on the etiopathogenic mechanisms underlying these diseases.

\section{Lymphedema}

The most frequent lymphatic anomaly is lymphedema. It is characterized by abnormal accumulation of interstitial fluid due to inefficient uptake and reduced flow, leading to swelling and disability, mostly in the extremities. Treatment is limited to pressotherapies, lymphatic drainage, and elastic compression and is never curative. Lymphedema can be primary (often congenital) or secondary (acquired; for example, after an infection or as a consequence of surgical breast cancer treatment).

Familial primary lymphedema commonly segregates in an autosomal dominant or recessive manner. It has been classified based on the age at onset into congenital (such as Nonne-Milroy lymphedema or type I lymphedema), peripubertal (hereditary lymphedema II, also known as Meige disease or lymphedema praecox), and late-onset lymphedema (onset after 35 years of age; also referred to as lymphedema tarda). Lymphedema can also occur in association with other clinical signs, as part of a syndrome. Nineteen genes have been identified as being mutated in different isolated or syndromic forms of lymphedema (Table 1). On the basis of these data, a complex algorithm for clinical diagnosis has been

Conflict of interest: The authors have declared that no conflict of interest exists. Citation for this article: J Clin Invest. 2014;124(3):898-904. doi:10.1172/JCI71614. proposed (2); however, the reduced penetrance of several of the signs and symptoms of the syndromes complicate its use. Interestingly, most of the proteins encoded by these genes seem to involve the VEGF-C/VEGFR-3 axis (3).

\section{VEGF-C/VEGFR-3 signaling axis}

The first mutations were discovered in FLT4, which encodes VEGFR-3 (Figure 1). Missense mutations in the tyrosine-kinase domain of the receptor cause primary congenital lymphedema (Nonne-Milroy lymphedema; OMIM 153100). This is usually present at birth, is bilateral, and affects the feet up to the knees $(4,5)$. Patients sometimes present with prenatal pleural effusion or in utero hydrops $(6,7)$. Nonne-Milroy lymphedema is usually an autosomal dominant disorder, yet de novo mutations are not infrequent (7-10). Thus, a family history of lymphedema is not required for diagnosis. Moreover, a family with a particular recessive VEGFR3 mutation has been reported (9). To date, mutations have been discovered in more than 100 families (Table 1 and refs. 3, 11).

Dominant VEGFR3 mutations inhibit receptor phosphorylation and prevent downstream signaling. The recessive mutation has a weaker effect, resulting in reduced ATP binding. In lymphoscintigraphy, hypoplasia or aplasia of the lymphatic system is observed (12). A similar phenotype is seen in the Chy mouse, which carries a spontaneous heterozygous point mutation in Vegfr 3 and is a model to study the pathophysiology and to perform preclinical trials of Nonne-Milroy lymphedema. The homozygous Vegfr3 knockout mice die around E9.5 due to irregular vessels with defective lumens $(13,14)$.

A mutation in the VEGFR-3 ligand, VEGFC, was reported in one family (Figure 1 and ref. 15). Clinically, these patients are indistinguishable from those with a FLT4 mutation. The predicted mutant protein was expressed in zebrafish but lacked detectable activity. Based on the position of the mutation (a small deletion in the fourth exon), the mutant allele likely undergoes nonsensemediated mRNA decay in human beings, resulting in negligible expression of the mutant protein. This would result in a loss of VEGF-C function. Similarly, spontaneous heterozygous Chy-3 mice, which develop chylous ascites and lymphedema, have a deletion of the whole VEGFC gene (16). Homozygotes lack all lymphatic vasculature and heterozygotes have lymphatic hypoplasia (17). Haploinsufficiency is therefore the most probable pathogenic mechanism for mutations in VEGFC. 


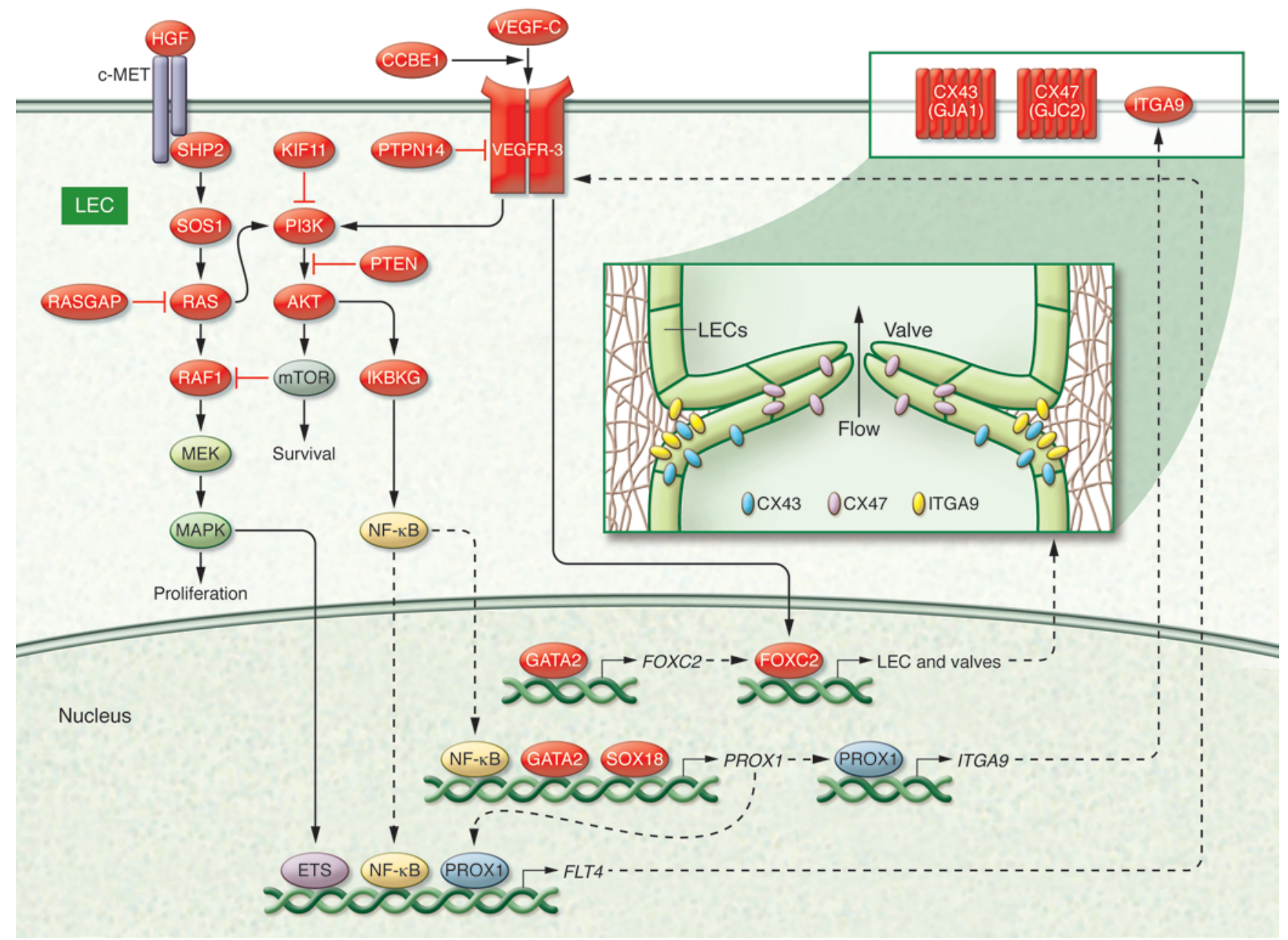

Figure 1

Schematic view of a lymphatic endothelial cell and valve. Lymphatic anomaly-associated proteins are frequently associated with the VEGF-C/ VEGFR-3 signaling pathway. Proteins mutated in lymphedema or other lymphatic disorders are shown in red. Arrows indicate direct or indirect interactions, red lines indicate inhibition, and dashed lines indicate transfer of protein. AKT includes AKT1; PIK3CA is part of PI3K; RAS includes HRAS and KRAS; RASA1 is one of the RASGAPs. LEC, lymphatic endothelial cell.

Collagen and calcium-binding EGF domain-containing protein 1 (CCBE1) binds to the extracellular matrix and potentiates the effects of VEGF-C on VEGFR-3 (Figure 1 and ref. 18). It is mutated in the zebrafish mutant full of fluid ( $f \circ f)$, where it was shown that CCBE1 is required for lymphangioblast budding and angiogenic sprouting from venous endothelium (19). In humans, homozygous or compound heterozygous mutations that abolish CCBE1 function cause highly penetrant, generalized lymphatic anomalies, including lymphedema and visceral lymphangiectasias (Table 1). These are associated with typical facial features and mental retardation, components of the Hennekam lymphangiectasia-lymphedema syndrome (OMIM 235510) (20-22).

PTPN14 is a protein tyrosine-phosphatase that is recruited to the VEGFR-3 receptor upon VEGF-C stimulation (Figure 1 and ref. 23). A homozygous deletion of exon 7 was found in a single consanguineous family with lymphedema and choanal atresia (OMIM 608911). The mutation results in a shift in the reading frame and the appearance of a premature termination codon (23), probably leading to nonsense-mediated mRNA decay and loss of function. Ptpn14-deficient mice mimic the human phenotype, as they develop lymphedema postnatally due to hyperplastic vessels (23). Thus, hyperactive VEGFR-3 signaling due to loss of the phosphatase also perturbs normal lymphatic development.

\section{Transcription factors}

Several transcription factors act downstream of VEGFR-3 (Figure 1). As they have pleiotropic effects through several target genes, their mutations cause syndromic forms of lymphedema. Truncating and missense mutations in FOXC2 are found in patients with late-onset lymphedema (hereditary lymphedema II; OMIM 153200), often associated with distichiasis (double row of eyelashes) and sometimes ptosis (OMIM 153400) and/or yellow nails (OMIM 153300) (Table 1 and refs. 24-30). Distichiasis has a high penetrance, yet not all patients with this feature carry a mutation in FOXC2. Foxc2 $2^{-1-}$ mice have abnormal lymphatic patterning and arrested lymphatic valve development. In heterozygotes, increased recruitment of pericytes hampers the function of collecting lymphatics, reminiscent of the human phenotype (31). 
Table 1

Genes involved in lymphatic anomalies

\begin{tabular}{|c|c|c|c|c|c|c|}
\hline Lymphatic anomalies/additional signs & Gene (protein) & Cases $^{A}$ & Penetrance ${ }^{B}$ & Mutation type & Inheritance & Animal models \\
\hline \multicolumn{7}{|l|}{ Isolated lymphedema } \\
\hline $\begin{array}{l}\text { Primary congenital lymphedema/ } \\
\text { Nonne-Milroy lymphedema }\end{array}$ & FLT4 (VEGFR-3) & $>100$ & High & Inactivating & $\begin{array}{l}\text { AD, AR, } \\
\text { de novo }\end{array}$ & Chy, Flt4 ${ }^{--}$ \\
\hline Milroy-like disease & VEGFC & 1 & High & LOF & $A D$ & Chy-3, Vegfc ${ }^{-1-}$ \\
\hline \multicolumn{7}{|l|}{ Syndromic lymphedema } \\
\hline $\begin{array}{l}\text { Hennekam lymphangiectasia-lymphedema } \\
\text { syndrome/mental retardation }\end{array}$ & CCBE1 & 13 & High & LOF & $\mathrm{AR}$ & fof \\
\hline $\begin{array}{l}\text { Lymphedema-distichiasis and yellow } \\
\text { nail syndromes/ptosis }\end{array}$ & FOXC2 & $>85$ & High & LOF & $A D$ & Foxc2 $2^{-1-}$ \\
\hline Hereditary lymphedema II (Meige disease) & GJC2 (CX47) & 7 & High & Missense & $A D$ & $\left(G j c 2^{-/-}\right)$ \\
\hline Oculodentodigital dysplasia/lymphedema & GJA1 (CX43) & 1 & High & Missense & $A D$ & $\left(G j a 1^{-/}\right)$ \\
\hline Choanal atresia/lymphedema & PTPN14 & 1 & High & LOF & $A R$ & Ptpn14-/- \\
\hline $\begin{array}{l}\text { Hypotrichosis-lymphedema-telangiectasia } \\
\text { syndrome }\end{array}$ & S0X18 & 3 & High & LOF?/D-N & $\begin{array}{l}\text { AR, } A D \text {, } \\
\text { de novo }\end{array}$ & $\begin{array}{l}\text { Ragged, } \\
(\text { Sox18- })\end{array}$ \\
\hline Lymphedema-lymphangiectasia & $H G F$ & 4 & Medium & LOF? & $A D ?$ & $\left(\mathrm{Met}^{--}\right)$ \\
\hline MCLMR & KIF11 & 14 & Low & LOF & AD, de novo & $\left(\right.$ Kif11 $\left.1^{-/}\right)$ \\
\hline $\begin{array}{l}\text { Noonan syndrome } 1 \\
\text { ( } 54 \% \text { with lymphedema) }\end{array}$ & PTPN11 (SHP2) & $>100$ & Medium & GOF & $A D$ & Shp2-- \\
\hline $\begin{array}{l}\text { Noonan syndrome } 1 \\
\text { (63\% with lymphedema) }\end{array}$ & SOS1 & Few with lymphedema & Medium & GOF & $A D$ & - \\
\hline $\begin{array}{l}\text { Primary lymphedema, myelodysplasia } \\
\text { (Emberger syndrome) }\end{array}$ & GATA2 & 13 & Low & LOF & $A D$ & $\left(\right.$ Gata2 $\left.^{--}\right)$ \\
\hline OLEDAID & IKBKG (NEMO) & 5 & Low & Hypomorphic & X-linked & $\left(\mathrm{lkbkg} \mathrm{g}^{--}\right)$ \\
\hline CM-AVM/lymphedema & RASA1 & Few with lymphedema & Low & LOF & $A D$ & $(\text { Rasa1-/- })^{\mathrm{D}}$ \\
\hline $\begin{array}{l}\text { Cholestasis-lymphedema syndrome } \\
\text { (Aagenaes syndrome) }\end{array}$ & locus in $15 q$ & - & - & - & $\mathrm{AR}$ & - \\
\hline \multicolumn{7}{|c|}{ Syndromic chylothorax/chylous ascites, lymphangiectasia } \\
\hline Fetal chylothorax & ITGA9 & 6 & High & Missense & AR, de novo & Itgag-- \\
\hline $\begin{array}{l}\text { Noonan syndrome, cardiofaciocutaneous } \\
\text { syndrome/chylothorax }\end{array}$ & KRAS & Few with chylothorax & Low & GOF & $A D$ & $\mathrm{Kras}^{--}$ \\
\hline Noonan syndrome 1/lymphangiectasia & RAF1 & Few with lymphangiectasia & Low & GOF & $A D$ & Raf1-KI \\
\hline $\begin{array}{l}\text { Costello syndrome/chylous ascites, } \\
\text { chylothorax }\end{array}$ & HRAS & $\begin{array}{l}\text { Few with chylous ascites/ } \\
\text { chylothorax }\end{array}$ & Low & GOF & $A D$ & Hras $^{-/}$ \\
\hline \multicolumn{7}{|l|}{ Syndromes with LMs } \\
\hline Turner syndrome/nuchal translucency & Monosomy X & $>100$ & Medium & - & Sex-linked & (X0 mice) \\
\hline $\begin{array}{l}\text { Proteus syndrome, Pten hamartoma } \\
\text { tumor syndrome }\end{array}$ & PTEN & $\sim 10$ & Medium & LOF & $A D$, de novo & $\left(\right.$ Pten $\left.^{--}\right)$ \\
\hline CLOVES, Klipple-Trenaunay-Weber syndrome & PIK3CA & 3 & $\mathrm{~N} / \mathrm{A}$ & GOF & Somatic & p $\alpha 100-K I$ \\
\hline Proteus syndrome & AKT1 & 40 & $\mathrm{~N} / \mathrm{A}$ & GOF & Somatic & Akt1-- \\
\hline
\end{tabular}

ANumber of index cases reported with a mutation. BPenetrance of lymphatic anomalies when carrying a mutation. ${ }^{\circ}$ Germline mutants only. Parentheses indicate the absence of lymphatic anomaly, and bolded text indicates that the model partially mimics the human phenotype. DInactivation in adults. AD, autosomal dominant; AR, autosomal recessive; D-N, dominant negative; GOF, gain of function; KI, knockin; LOF, loss of function; -, not applicable. Question marks indicate that mutation type and/or inheritance is unclear.

VEGFR-3 expression is controlled by PROX1, a crucial transcription factor for initiation of lymphangiogenesis. PROX1 is under the control of another transcription factor, SOX18 (Figure 1). The latter is mutated in the rare hypotrichosis-lymphedema-telangiectasia syndrome (OMIM 607823), which is characterized by variable-onset lymphedema associated with sparse hair and cutaneous telangiectasias. A dominant nonsense mutation is located in the transactivation domain of SOX18 and two recessive substitutions in the DNA-binding domain (32). The former may compete for DNA binding without transcriptional activation of target genes, whereas the latter likely have less affinity to their promoter binding motifs. Ragged mice, which have Sox 18 mutations, are phenotypically similar $(32,33)$. Sox18 is expressed in endothelial cells, hair and feather follicles, and the heart (34), but no cardiac phenotype is seen in patients or mice. The Sox18 knockout mice have only a mild coat defect (35). This is likely due to redundancy with two close homologs, SOX7 and SOX17. The human mutations may therefore have dominant-negative effects, via competitive transcription factor binding (32). 
GATA2 is a transcription factor that controls PROX1 and FOXC2 expression (Figure 1). Most GATA2 mutations are associated with dendritic cell, monocyte, B lymphocyte, and natural killer lymphocyte deficiency (OMIM 614172), with susceptibility to myelodysplastic syndrome (OMIM 614286) and acute myeloid leukemia (OMIM 601626). However, some GATA2 mutations have been discovered in patients with primary lymphedema with myelodysplasia (Emberger syndrome; OMIM 614038), which is characterized by lymphedema and myelodysplasia $(36,37)$. There is no clear phenotype-genotype correlation, suggesting that modifiers play a role.

Besides cross-regulation, these transcription factors modify expression levels of several other proteins, some of which are involved in lymphangiogenesis (Figure 1). PROX1 regulates FLT4, and FOXC2 controls proteins essential for lymphatic valves, such as connexins. Thus, adequate spatiotemporal control of their activity is essential for proper lymphatic vessel development and function.

\section{Nuclear and transcription factor regulators}

Mutations in IKBKG and KIF11, which modulate the activity of transcription factors or affect nuclear dynamics, cause complex lymphedema syndromes. IKBKG (also known as NEMO) is an NF-KB modulator. It is mutated in patients with the rare X-linked syndrome anhydrotic ectodermal dysplasia with immunodeficiency, osteopetrosis, and lymphedema (OLEDAID; OMIM 300301), associated with incontinentia pigmenti in the mother (OMIM 308300) (Figure 1). Five cases of OLEDAID with an IKBKG mutation have been reported (Table 1 and refs. 38-41). Loss-of-function mutations cause incontinentia pigmenti, which is embryonic lethal in males and in homozygous $\mathrm{Ik}_{\mathrm{kg}} /$ - murine females, who die from severe apoptosis (42). The OLEDAID-causing mutations are hypomorphs, which diminish but do not abolish the ability of IKBKG to activate

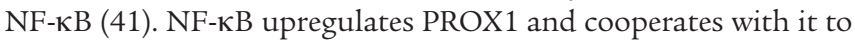
induce VEGFR-3 expression (Figure 1 and ref. 43).

KIF11 encodes EG5, which acts as a homotetrameric kinesin motor. Members of this protein family are involved in establishing a bipolar spindle during mitosis for chromosome positioning and centrosome separation. Heterozygous KIF11 mutations cause a specific syndrome consisting of lower limb lymphedema of variable expressivity associated with microcephaly with or without chorioretinopathy, lymphedema, or mental retardation (MCLMR; OMIM 152950) (44). The EG5 mutations causing MCLMR may have dominant-negative effects, since $\mathrm{Kifl}^{+/-}$mice are phenotypically normal and $\mathrm{Kif1}^{-/-}$mice die prior to implantation (45). A link to the VEGF-C/VEGFR-3 pathway has not been established, but inhibition of EG5 activates the PI3K/AKT pathway (Figure 1 and ref. 46).

\section{Connexins}

The transcription factors involved in VEGF-C/VEGFR-3 signaling regulate expression of genes essential for lymphatic (and venous) valve development and maintenance. These include the gap junction proteins, connexins (Figure 1 and ref. 47). Mutations in two connexins have been identified in a few lymphedema patients (Table 1). They consist of amino acid substitutions that alter but do not abolish connexin function.

Substitutions of highly conserved amino acids in connexin 47 (CX47), encoded by GJC2, cause lymphedema in all four extremities $(48,49)$. In contrast, loss-of-function mutations, primarily premature stop codons, cause hypomyelinating leukodystrophy 2 (OMIM 608804), in which lymphedema does not occur. CX47 is expressed in lymphatic endothelial cells, on the upstream side of lymphatic valves (Figure 1 and ref. 47). The amino acid substitutions may have gain-of-function effects, as $C x 47$ homozygous knockouts have no lymphatic defect (Table 1 and ref. 50).

An amino acid substitution in another connexin, CX43 (GJA1), can also cause lymphedema, as part of the oculodentodigital dysplasia (OMIM 164200), which affects the eyes, face, teeth, and digits. (51). CX43 is also highly enriched on the upstream side of lymphatic valves (Figure 1), where it forms hemi- and intercellular channels (47). The only GJA1 mutation reported so far to cause lymphedema likely specifically alters channel properties leading to valve dysfunction. Homozygous inactivation of $C x 43$ in mice is lethal at birth because of cardiac malformation (52).

\section{Rasopathies and lymphedema}

Lymphedema, chylothorax, or chylous ascites are also variably seen in patients with mutations in genes of the RAS signaling pathway, commonly referred to as rasopathies. Noonan syndrome 1 (OMIM 163950 ) is caused by germline mutations in PTPN11 encoding SHP2 (53), a mediator of tyrosine kinase receptor signaling (Figure 1), in $50 \%$ of patients. In another $13 \%$ of patients, the mutation is in SOS1 (53). Lymphedema is present in half of the patients with mutations in PTPN11, and in over $60 \%$ of those with a SOS1 mutation (Table 1 and ref. 54).

In some patients, activating mutations associated with lymphedema or lymphangiectasia are found in KRAS (55) or RAF1 (56). KRAS usually causes the cardiofaciocutaneous syndrome (57), and mutations in HRAS are associated with Costello syndrome $(58,59)$. In both disorders, chylothorax and chylous ascites can be seen. Such features have also been observed in a small number of patients with a RASA1 mutation causing capillary malformation-arteriovenous malformation (CM-AVM) syndrome $(60,61)$. RASA1 encodes the RAS GTPase p120RASGAP (Figure 1), and inactivation of this gene in adult mice results in alteration of the lymphatic system (62). All these anomalies lead to increased RAS pathway activity.

\section{Additional genes}

In addition to the above-mentioned genes that are known to carry mutations in subgroups of patients with variable penetrance of lymphedema (Table 1), several other genes may be involved in lymphatic anomalies. A recurrent integrin $\alpha 9$ (ITGA9) missense mutation seems to associate with severe chylothorax in human fetuses (63-65). ITGA9 expression is controlled by PROX1 (66). ITGA9 binds to fibronectin and is essential for the development of lymphatic valves (Figure 1) (67). Inactivation in mice results in fatal bilateral chylothorax (68).

Alterations in hepatocyte growth factor $(H G F)$ and its receptor $(M E T)$ were reported in a few families with lymphedema (69). However, bioinformatic re-analysis of the MET changes predicted that these mutations are benign. The four HGF mutations, two of which are premature stop codons, are probably damaging. HGF is an interesting predisposing factor, as it stimulates lymphangiogenesis in vitro and in vivo (70). Confirmatory genetic data are needed.

Cholestasis-lymphedema syndrome (OMIM 214900), also known as Aagenaes syndrome, has been linked to chromosome 15 on the basis of haplotype sharing (71). The causative gene is unknown. A CCBE1 mutation was also found in one patient with cholestasis-lymphedema syndrome, suggesting locus heterogeneity (72).

Overall, genetic mutations in a subset of eight genes explain the etiology of about $40 \%$ of familial lymphedema cases and $10 \%$ of the sporadic cases (ref. 3 and our unpublished observations). 
This comprises less than $25 \%$ of patients with lymphedema. Some of the remaining heritability could be due to mutations in the other 11 known genes, but such mutations are likely rare, since the syndromes caused by mutations in those genes are particular and well characterized and/or the penetrance of lymphedema is low. Although genetic screens are rarely, if ever, exhaustive, additional genes that are mutated in primary lymphedema are highly likely to exist.

\section{Secondary lymphedema}

Secondary lymphedema is induced by external stimuli, such as infection, surgery, or radiotherapy. It may be influenced by genetic predisposition (73). Predisposing changes were reported in the MET gene (69) and in the GJC2 gene (74). Changes in the latter were found to increase risk for developing secondary lymphedema following breast cancer treatment.

Secondary lymphedema often develops years after an invasive treatment. Dissection of its genetic predisposition therefore requires long-term prospective studies. Lymphangiogenesis is under the control of a tightly orchestrated equilibrium between numerous players. Predisposition to secondary lymphedema may be based on weak changes in a number of these genes instead of strong, mutation-like changes. Their identification would help develop novel, targeted therapeutic modalities.

\section{LMs}

LMs are localized lesions that consist of dilated lymphatic channels filled with lymph but disconnected from the normal lymphatic system (75). LMs are congenital and enlarge when infected. They are sporadic, usually unifocal, and their etiopathogenesis is unknown. The lack of familial forms and the unifocality of the lesions suggest that the cause could be a somatic mutation, restricted to cells of the lesion, and that would be too damaging (i.e., lethal) if occurring germline. Such a scenario held true for sporadic venous malformations, which we have demonstrated to result from strongly activating somatic mutations in TIE2 $(76,77)$. Other developmental disorders have since been shown to be due to similar post-zygotic mosaic mutations $(78,79)$.

LMs can be part of a syndrome, such as Turner syndrome (due to monosomy $\mathrm{X}$ ), or overgrowth syndromes, such as proteus syndrome (OMIM 176920), Klippel-Trenaunay-Weber syndrome (capillary-lymphatico-venous malformation; OMIM 149000), and CLOVES syndrome (OMIM 612918) due to mutations in the PI3K/AKT pathway (Figure 1 and Table 1). PTEN mutations are found in a subset of patients with PTEN hamartoma tumor syndrome; some are inherited (80). In contrast, a recurrent somatic activating mutation was discovered in $A K T 1$ in patients with pro-

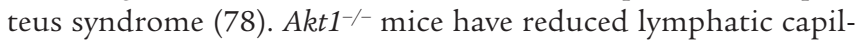
laries and valve numbers, underscoring an important function in lymphangiogenesis (81). Somatic mutations that activate PIK3CA were identified in patients with CLOVES and some with Klippel-Trenaunay-Weber syndrome (79). All of these proteins are core elements of the PI3K/AKT/mTOR pathway (Figure 1), suggesting that other overgrowth syndromes as well as sporadic LMs may be caused by somatic changes in the components of this pathway.

\section{Current methods and future directions}

Mutations in the VEGF-C/VEGFR-3 axis are a central mechanism in the etiopathogenesis of inherited lymphedema (Figure 1). Many of the mutated genes encode proteins involved in the downstream regulation of target gene expression or proteins of the RAS/ MAPK pathway that regulate VEGFR-3 expression. Yet the cause of primary lymphedema remains unexplained for many patients. Some syndromes with localized lymphatic dysplasia are caused by somatic mutations that tend to cluster around PI3K/AKT signaling. There are connections between the two pathways (Figure 1), suggesting that the etiopathogenic causes of primary (and secondary) lymphedema may cluster around these intracellular functions.

For many years linkage analysis and positional cloning have been used to identify disease-causing genetic mutations, as was the case for VEGFR 3 and FOXC2. In some instances, for example SOX18 and CCBE1, this approach was helped by the identification of spontaneous mutant animal models that guided the human genetic studies. In other cases, researchers utilized autozygosity mapping in large consanguineous families (PTPN14). More recently, the advent of next-generation sequencing has allowed analysis of large candidate regions or the whole exome (whole-exome sequencing [WES]). Using WES on several patients with a similar phenotype led to the identification of mutations in novel genes, such as KIF11 and VEGFC. In the latter, restricting the analysis to candidate genes proved successful.

The unexplained lymphatic anomalies might also result from a combination of mildly mutated germline alleles that are more difficult to identify and mutations in regulatory regions and/or epigenetic changes. The latter two are not detected by WES. Epigenome and whole-exome sequencing may thus be indicated in the future. However, within primary lymphedema, in which locus heterogeneity is high and sporadic cases are not rare, targeted high-throughput sequencing of a panel of genes has become the method of choice for diagnostic screens.

It is difficult to treat lymphedema, whether primary or secondary. Genetic insights point toward VEGF-C/VEGFR-3 signaling as a target. Autologous grafts are currently being tested in combination with adenoviral expression of VEGF-C in preclinical studies of secondary lymphedema (82). This seems to increase lymphangiogenesis around the implanted lymph nodes, helping them to connect with the lymphatic system. Another candidate is HGF. Injection of a plasmid expressing HGF in rat tail (83) or in a mouse model of upper limb lymphedema that simulates breast cancer-related lymphedema (84) prevented overt development of lymphedema and stimulated development of new lymphatic vessels in the long term. Such growth factors may potentially be useful in inducing the development functional lymphatics.

The RAS/MAPK pathway is activated in some forms of syndromic lymphedema (Noonan syndrome) and CM-AVM syndrome. Many inhibitors of this pathway are being developed in laboratories and tested in preclinical trials for cancer therapy. Some of them may be applicable as therapies for syndromic lymphedema.

In regard to syndromes with germline or somatic mutations activating the PI3K/AKT signaling pathway, small-molecule inhibitors may become useful as treatment. Many such inhibitors exist, and some are in clinical use for cancer, including the mTOR inhibitor rapamycin. Preliminary results on PTEN lesions and lymphatic anomalies are encouraging $(85,86)$.

The role of genetics in lymphatic anomalies is complex, as illustrated by the 23 mutated human genes reported thus far (Table 1). Their respective prevalence has not been studied in detail on large series of patients, and the lymphatic defect is sometimes a rare or minor feature of the syndromic phenotype. With the increased availability of high-throughput sequencing on large sets of 
patients with lymphatic defects, several additional genes will likely be revealed. The clarification of the defective signaling pathways will also allow the generation of better animal models to test new molecules as targeted therapies to treat lymphatic anomalies.

\section{Acknowledgments}

The studies mentioned were partially supported by funding from the Belgian Federal Science Policy Office Interuniversity Attraction Poles (BELSPO-IAP) program through the project
IAP P7/43-BeMGI, and by the Fonds de la Recherche Scientifique (F.R.S.-FNRS). We also acknowledge the support of the Fédération Wallonie-Bruxelles and the Loterie Nationale of Belgium. We thank Liliana Niculescu for secretarial help.

Address correspondence to: Miikka Vikkula, Laboratory of Human Molecular Genetics, Avenue Hippocrate, 74, B-1200 Brussels, Belgium. Phone: 32.2.7647490; Fax: 32.2.7647460; E-mail: miikka. vikkula@uclouvain.be.
1. Mulliken JB, Burrows PE, Fishman SJ, eds. Mulliken and Young's Vascular Anomalies, Hemangiomas and Malformations. 2nd ed. New York, New York, USA: Oxford University Press; 2013.

2. Connell F, et al. The classification and diagnostic algorithm for primary lymphatic dysplasia: an update from 2010 to include molecular findings. Clin Genet. 2013;84(4):303-314.

3. Mendola A, et al. Mutations in the VEGFR3 signaling pathway explain $36 \%$ of familial lymphedema. Mol Syndromol. 2013;4(6):257-266.

4. Irrthum A, Karkkainen MJ, Devriendt K, Alitalo $\mathrm{K}$, Vikkula M. Congenital hereditary lymphedema caused by a mutation that inactivates VEGFR3 tyrosine kinase. Am J Hum Genet. 2000;67(2):295-301.

5. Karkkainen MJ, et al. Missense mutations interfere with VEGFR-3 signalling in primary lymphoedema. Nat Genet. 2000;25(2):153-159.

6. Daniel-Spiegel E, et al. Hydrops fetalis: an unusual prenatal presentation of hereditary congenital lymphedema. Prenat Diagn. 2005;25(11):1015-1018.

7. Ghalamkarpour A, et al. Hereditary lymphedema type I associated with VEGFR3 mutation: the first de novo case and atypical presentations. Clin Genet. 2006;70(4):330-335.

8. Ghalamkarpour A, et al. Sporadic in utero generalized edema caused by mutations in the lymphangiogenic genes VEGFR3 and FOXC2.J Pediatr. 2009; 155(1):90-93.

9. Ghalamkarpour A, et al. Recessive primary congenital lymphoedema caused by a VEGFR3 mutation. J Med Genet. 2009;46(6):399-404.

10. Carver C, Brice G, Mansour S, Ostergaard P, Mortimer P, Jeffery S. Three children with Milroy disease and de novo mutations in VEGFR3. Clin Genet. 2007;71(2):187-189.

11. Gordon K, et al. FLT4/VEGFR3 and Milroy disease: novel mutations, a review of published variants and database update. Hum Mutat. 2013;34(1):23-31.

12. Connell F, Brice G, Mortimer P. Phenotypic characterization of primary lymphedema. Ann NY Acad Sci. 2008;1131:140-146.

13. Karkkainen MJ, et al. A model for gene therapy of human hereditary lymphedema. Proc Natl Acad Sci US A. 2001;98(22):12677-12682.

14. Dumont DJ, et al. Cardiovascular failure in mouse embryos deficient in VEGF receptor-3. Science. 1998;282(5390):946-949.

15. Gordon K, et al. Mutation in vascular endothelial growth factor-C, a ligand for vascular endothelial growth factor receptor-3, is associated with autosomal dominant milroy-like primary lymphedema. Circ Res. 2013;112(6):956-960.

16. Dellinger MT, Hunter RJ, Bernas MJ, Witte MH, Erickson RP. Chy-3 mice are Vegfc haploinsufficient and exhibit defective dermal superficial to deep lymphatic transition and dermal lymphatic hypoplasia. Dev Dyn. 2007;236(8):2346-2355.

17. Karkkainen MJ, et al. Vascular endothelial growth factor $\mathrm{C}$ is required for sprouting of the first lymphatic vessels from embryonic veins. Nat Immunol. 2004;5(1):74-80.

18. Bos FL, et al. CCBE1 is essential for mammalian lymphatic vascular development and enhances the lymphangiogenic effect of vascular endothelial growth factor-C in vivo. Circ Res. 2011;109(5):486-491.

19. Hogan BM, et al. Ccbe1 is required for embryonic lymphangiogenesis and venous sprouting. Nat Genet. 2009;41(4):396-398.

20. Alders M, et al. Evaluation of clinical manifestations in patients with severe lymphedema with and without CCBE1 mutations. Mol Syndromol. 2013;4(3):107-113

21. Alders M, et al. Mutations in CCBE1 cause generalized lymph vessel dysplasia in humans. Nat Genet. 2009;41(12):1272-1274.

22. Connell F, et al. Linkage and sequence analysis indicate that CCBE1 is mutated in recessively inherited generalised lymphatic dysplasia. Hum Genet. 2010;127(2):231-241.

23. Au AC, et al. Protein tyrosine phosphatase PTPN14 is a regulator of lymphatic function and choanal development in humans. Am J Hum Genet. 2010;87(3):436-444.

24. Bell R, et al. Analysis of lymphoedema-distichiasis families for FOXC2 mutations reveals small insertions and deletions throughout the gene. Hum Genet. 2001;108(6):546-551.

25. Fang J, et al. Mutations in FOXC2 (MFH-1), a forkhead family transcription factor, are responsible for the hereditary lymphedema-distichiasis syndrome. Am J Hum Genet. 2000;67(6):1382-1388.

26. Finegold DN, et al. Truncating mutations in FOXC2 cause multiple lymphedema syndromes. Hum Mol Genet. 2001;10(11):1185-1189.

27. Dagenais SL, Hartsough RL, Erickson RP, Witte MH, Butler MG, Glover TW. Foxc2 is expressed in developing lymphatic vessels and other tissues associated with lymphedema-distichiasis syndrome. Gene Expr Patterns. 2004;4(6):611-619.

28. van Steensel MA, et al. Novel missense mutations in the FOXC2 gene alter transcriptional activity. Hum Mutat. 2009;30(12):E1002-E1009.

29. Fauret AL, Tuleja E, Jeunemaitre X, Vignes S. A novel missense mutation and two microrearrangements in the FOXC2 gene of three families with lymphedema-distichiasis syndrome. Lymphology. 2010;43(1):14-18.

30. Witte MH, et al. Lymphedema-distichiasis syndrome without FOXC2 mutation: evidence for chromosome 16 duplication upstream of FOXC2. Lymphology. 2009;42(4):152-160.

31. Petrova TV, et al. Defective valves and abnormal mural cell recruitment underlie lymphatic vascular failure in lymphedema distichiasis. Nat Med. 2004; 10(9):974-981.

32. Irrthum A, et al. Mutations in the transcription factor gene SOX18 underlie recessive and dominant forms of hypotrichosis-lymphedema-telangiectasia. Am J Hum Genet. 2003;72(6):1470-1478.

33. Pennisi D, et al. Mutations in Sox18 underlie cardiovascular and hair follicle defects in ragged mice. Nat Genet. 2000;24(4):434-437.

34. Pennisi DJ, James KM, Hosking B, Muscat GE, Koopman P. Structure, mapping, and expression of human SOX18. Mamm Genome. 2000;11(12):1147-1149.

35. Pennisi D, Bowles J, Nagy A, Muscat G, Koopman P. Mice null for sox18 are viable and display a mild coat defect. Mol Cell Biol. 2000;20(24):9331-9336.

36. Kazenwadel J, et al. Loss-of-function germline
GATA2 mutations in patients with MDS/AML or MonoMAC syndrome and primary lymphedema reveal a key role for GATA2 in the lymphatic vasculature. Blood. 2012;119(5):1283-1291.

37. Ostergaard P, et al. Mutations in GATA2 cause primary lymphedema associated with a predisposition to acute myeloid leukemia (Emberger syndrome). Nat Genet. 2011;43(10):929-931.

38. Doffinger R, et al. X-linked anhidrotic ectodermal dysplasia with immunodeficiency is caused by impaired NF-КB signaling. Nat Genet. 2001; 27(3):277-285.

39. Smahi A, et al. Genomic rearrangement in NEMO impairs NF- $\kappa \mathrm{B}$ activation and is a cause of incontinentia pigmenti. The International Incontinentia Pigmenti (IP) Consortium. Nature. 2000; 405(6785):466-472.

40. Roberts CM, Angus JE, Leach IH, McDermott EM, Walker DA, Ravenscroft JC. A novel NEMO gene mutation causing osteopetrosis, lymphoedema, hypohidrotic ectodermal dysplasia and immunodeficiency (OL-HED-ID). Eur J Pediatr. 2010; 169(11):1403-1407.

41. Carlberg VM, et al. Hypohidrotic ectodermal dysplasia, osteopetrosis, lymphedema, and immunodeficiency in an infant with multiple opportunistic infections [published online ahead of print February 14, 2013]. Pediatr Dermatol. doi:10.1111/pde.12103.

42. Schmidt-Supprian M, et al. NEMO/IKK $\gamma$-deficient mice model incontinentia pigmenti. Mol Cell. 2000;5(6):981-992.

43. Flister MJ, et al. Inflammation induces lymphangiogenesis through up-regulation of VEGFR-3 mediated by NF-KB and Prox1. Blood. 2010;115(2):418-429.

44. Ostergaard P, et al. Mutations in KIF11 cause autosomal-dominant microcephaly variably associated with congenital lymphedema and chorioretinopathy. Am J Hum Genet. 2012;90(2):356-362.

45. Chauviere M, Kress C, Kress M. Disruption of the mitotic kinesin Eg5 gene (Knsl1) results in early embryonic lethality. Biochem Biophys Res Commun. 2008;372(4):513-519.

46. Liu M, et al. Inhibition of the mitotic kinesin Eg5 up-regulates Hsp70 through the phosphatidylinositol 3-kinase/Akt pathway in multiple myeloma cells. J Biol Chem. 2006;281(26):18090-18097.

47. Kanady JD, Simon AM. Lymphatic communication: connexin junction, what's your function? Lymphology. 2011;44(3):95-102.

48. Ferrell RE, et al. GJC2 missense mutations cause human lymphedema. Am J Hum Genet. 2010; 86(6):943-948.

49. Ostergaard P, et al. Rapid identification of mutations in GJC2 in primary lymphoedema using whole exome sequencing combined with linkage analysis with delineation of the phenotype. J Med Genet. 2011;48(4):251-255

50. Tress $\mathrm{O}$, et al. Pathologic and phenotypic alterations in a mouse expressing a connexin 47 missense mutation that causes Pelizaeus-Merzbacher-like disease in humans. PLoS Genet. 2011;7(7):e1002146.

51. Brice G, Ostergaard P, Jeffery S, Gordon K, Mortimer P, Mansour S. A novel mutation in GJA1 causing oculodentodigital syndrome and primary lymphoedema in a three generation family. Clin 
Genet. 2013;84(4):378-381.

52. Reaume AG, et al. Cardiac malformation in neonatal mice lacking connexin43. Science. 1995 267(5205):1831-1834.

53. Tartaglia M, Zampino G, Gelb BD. Noonan syndrome: clinical aspects and molecular pathogenesis. Mol Syndromol. 2010;1(1):2-26.

54. Smpokou P, Tworog-Dube E, Kucherlapati RS, Roberts AE. Medical complications, clinical findings, and educational outcomes in adults with Noonan syndrome. Am JMed Genet A. 2012;158A(12):3106-3111.

55. de Mooij YM, van den Akker NM, Bekker MN, Bartelings MM, van Vugt JM, Gittenberger-de Groot AC. Aberrant lymphatic development in euploid fetuses with increased nuchal translucency including Noonan syndrome. Prenat Diagn. 2011;31(2):159-166.

56. Lee WJ, et al. Novel occurrence of microcystic lymphatic malformation and linear port-wine stain in patients with Noonan syndrome. Int J Dermatol. 2010;49(2):232-233.

57. Schubbert S, et al. Germline KRAS mutations cause Noonan syndrome. Nat Genet. 2006;38(3):331-336

58. Kerr B, et al. Genotype-phenotype correlation in Costello syndrome: HRAS mutation analysis in 43 cases. J Med Genet. 2006;43(5):401-405.

59. Lo IF, et al. Severe neonatal manifestations of Costello syndrome. J Med Genet. 2008;45(3):167-171.

60 . Revencu N, et al. RASA1 mutations and associated phenotypes in 68 families with capillary malformation-arteriovenous malformation. Hum Mutat 2013;34(12):1632-1641.

61. Burrows PE, et al. Lymphatic abnormalities are associated with RASA1 gene mutations in mouse and man. Proc Natl Acad Sci U S A. 2013;110(21):8621-8626.

62. Lapinski PE, et al. RASA1 maintains the lymphatic vasculature in a quiescent functional state in mice. J Clin Invest. 2012;122(2):733-747.

63. Ma GC, et al. A recurrent ITGA9 missense mutation in human fetuses with severe chylothorax: possible correlation with poor response to fetal therapy. Prenat Diagn. 2008;28(11):1057-1063.
64. Yeang $\mathrm{CH}$, et al. Genome-wide gene expression analysis implicates the immune response and lymphangiogenesis in the pathogenesis of fetal chylothorax. PLoS One. 2012;7(4):e34901.

65. Yang YS, et al. Experimental treatment of bilateral fetal chylothorax using in-utero pleurodesis. Ultrasound Obstet Gynecol. 2012;39(1):56-62.

66. Mishima K, et al. Prox1 induces lymphatic endothelial differentiation via integrin $\alpha 9$ and other signaling cascades. Mol Biol Cell. 2007;18(4):1421-1429.

67 . Bazigou E, et al. Integrin- $\alpha 9$ is required for fibronectin matrix assembly during lymphatic valve morphogenesis. Dev Cell. 2009;17(2):175-186

68. Huang XZ, et al. Fatal bilateral chylothorax in mice lacking the integrin $\alpha 9 \beta 1$. Mol Cell Biol. 2000;20(14):5208-5215

69. Finegold DN, et al. HGF and MET mutations in primary and secondary lymphedema. Lymphat Res Biol. 2008;6(2):65-68.

70. Kajiya K, Hirakawa S, Ma B, Drinnenberg I, Detmar M. Hepatocyte growth factor promotes lymphatic vessel formation and function. EMBO J. 2005;24(16):2885-2895

71. Bull LN, et al. Mapping of the locus for cholestasis-lymphedema syndrome (Aagenaes syndrome) to a 6.6-cM interval on chromosome 15q. Am J Hum Genet. 2000;67(4):994-999.

72. Shah S, et al. Mutation in two siblings, one manifesting lymphedema-cholestasis syndrome, and the other, fetal hydrops. PLoS One. 2013;8(9):e75770.

73. Newman B, et al. Possible genetic predisposition to lymphedema after breast cancer. Lymphat Res Biol. 2012;10(1):2-13.

74. Finegold DN, et al. Connexin 47 mutations increase risk for secondary lymphedema following breast cancer treatment. Clin Cancer Res. 2012;18(8):2382-2390.

75. Boon LM, Vikkula M. Vascular anomalies. In: Wolff K, Goldsmith LA, Katz LI, Gilchrest BA, Paller AS, Leffell DJ, eds. Fitzpatrick's Dermatology in General Med icine. 7th ed. New York, New York, USA: McGraw-Hill Professional Publishing; 2008:1651-1666.
76. Limaye N, et al. Somatic mutations in angiopoietin receptor gene TEK cause solitary and multiple sporadic venous malformations. Nat Genet. 2009; 41(1):118-124

77. Soblet J, Limaye N, Uebelhoer M, Boon LM, Vikkula M. Variable somatic TIE2 mutations in half of sporadic venous malformations. Mol Syndromol. 2013;4(4):179-183

78. Lindhurst MJ, et al. A mosaic activating mutation in AKT1 associated with the Proteus syndrome. N Engl J Med. 2011;365(7):611-619.

79. Kurek KC, et al. Somatic mosaic activating mutations in PIK3CA cause CLOVES syndrome. Am J Hum Genet. 2012;90(6):1108-1115.

80. Zhou XP, Marsh DJ, Hampel H, Mulliken JB, Gimm O, Eng C. Germline and germline mosaic PTEN mutations associated with a Proteus-like syndrome of hemihypertrophy, lower limb asymmetry, arteriovenous malformations and lipomatosis. Hum Mol Genet. 2000;9(5):765-768.

81. Zhou F, et al. Akt/Protein kinase B is required for lymphatic network formation, remodeling, and valve development. Am J Pathol. 2010;177(4):2124-2133.

82. Honkonen KM, et al. Lymph node transfer and perinodal lymphatic growth factor treatment for lymphedema. Ann Surg. 2013;257(5):961-967.

83. Saito Y, et al. Transfection of human hepatocyte growth factor gene ameliorates secondary lymphedema via promotion of lymphangiogenesis. Circulation. 2006;114(11):1177-1184.

84. Saito Y, Nakagami H, Kaneda Y, Morishita R. Lymphedema and therapeutic lymphangiogenesis. Biomed Res Int. 2013;2013:804675.

85. Iacobas I, Burrows PE, Adams DM, Sutton VR, Hollier LH, Chintagumpala MM. Oral rapamycin in the treatment of patients with hamartoma syndromes and PTEN mutation. Pediatr Blood Cancer. 2011;57(2):321-323

86. Hammill AM, et al. Sirolimus for the treatment of complicated vascular anomalies in children. Pediatr Blood Cancer. 2011;57(6):1018-1024. 